\title{
Análisis de las complicaciones de los catéteres permanentes para hemodiálisis en un área de salud: repercusión económica
}

\author{
Maria Pilar Velayos González*\# - Susana Martínez Gómez*\#\# - José Portolés Pérez**\# - Mª del Carmen \\ Gago Gómez*\# - Ma del Mar Andrés Vázquez*\#\# - Enrique Gruss Vergara**\#
}

\author{
* Enfermera \\ ** Médico \\ \# Fundación Hospital de Alcorcón \\ \#\# Fundación Íñigo Alvarez de Toledo: "Los Llanos"
}

\section{Resumen}

En los últimos años han aumentado los pacientes portadores de catéter permanente para hemodiálisis.

Objetivos: Recoger complicaciones asociadas a los catéteres estimando su coste global. Comparar el coste de la conexión y desconexión de catéter versus fístula.

Material y métodos: Estudio prospectivo descriptivo de 24 meses, en 116 CT para HD en 97 pacientes: $68,05 \%$ hombres, edad media 66,56 años y $23,3 \%$ diabéticos.

Resultados: Siguen funcionando 41 catéteres, se retiraron 75 por: malfunción (11), uso fístula (37), paso a diálisis peritoneal (9), infección (6) y

\begin{tabular}{|c|}
\hline Correspondencia: \\
Susana Martínez Gómez \\
Fundación Hospital de Alcorcón \\
C. Budapest, n 1 \\
28922 Alcorcón. Madrid \\
sumgomez@yahoo.es \\
\hline
\end{tabular}

otros (12). En 59 ocasiones se utilizó urokinasa por bajo flujo, se solucionaron 45 y se revisaron 14 en radiología vascular, debiendo sustituirse (6). Registramos 8 infecciones sistémicas, 7 del orificio de salida y 3 del túnel. La supervivencia media acumulada de los catéteres fue de $611,70 \pm 36,76$ días. La supervivencia fue menor en insuficiencia renal de origen vascular $293,17 \pm 46,9$. El gasto total de los catéteres fue de 203.354,07€. El coste mes/catéter fue de $563,26 €$. El coste por sesión sin complicaciones de fue de15,66€ en catéter y de 6,376€ en fístulas.

Conclusiones 1. Nuestros resultados en supervivencia y complicaciones mejoran los recogidos en las guías clínicas. 2. El uso y mantenimiento de los catéteres genera un gasto importante y su uso supone el doble de gasto que la fístula.

\section{PALABRAS CLAVE:}

- CATÉTER

- COMPLICACIONES

- COSTES

- HEMODIÁLISIS 


\section{Analysis of complication of permanent catheters for haemodialysis in a health area: economic repercussions}

\section{Abstract}

In recent years the number of patients with a permanent catheter for haemodialysis has increased.

Aims: To compile data on the complications associated to catheters, estimating the overall cost. To compare catheter connection and disconnection costs with the cost of a fistula.

Material and methods: A 24-month prospective descriptive study in 116 CT for HD in 97 patients: $68.05 \%$ men, average age 66.56 years and $23.3 \%$ diabetic.

Results: A total of 41 catheters continue to work, 75 were removed because of: malfunction (11), use of fistula (37), transfer to peritoneal dialysis (9), infection (6) and others (12). On 59 occasions urokinase was used for low flow, 45 cases were solved and 14 were reviewed in vascular radiology, with 6 having to be replaced. We recorded 8 systemic infections, 7 of the catheter wound and 3 of the tunnel. The average aggregate survival of the catheters was $611.70 \pm 36.76$ days. Survival was lower in renal insufficiency of vascular origin 293.17 \pm 46.9 . The total expense of the catheters was $203,354.07 €$. The cost month/catheter was $563.26 €$. The cost per session without complications was $15.66 €$ in catheters and $6.376 €$ in fistulas.

Conclusions: 1. Our results in survival and complications improve on those indicated in clinical guidelines. 2. The use and maintenance of catheters generates a significant expense and their use involves double the expense of a fistula.

\section{KEY WORDS}

- CATHETER

- COMPLICATIONS

- COSTS

- HAEMODIALYSIS

\section{Introducción}

El perfil del paciente renal que recibe hemodiálisis (HD) como tratamiento sustitutivo ha cambiado. En los últimos años hemos asistido a un aumento de los pacientes portadores de un catéter permanente 0 temporal como acceso vascular (AV) ${ }^{1}$. El aumento de su uso hace que las complicaciones relacionadas con este tipo de AV constituyan un problema actual y creciente en la asistencia al paciente en HD $^{2}$.

La actitud de los profesionales sanitarios que forman los equipos multidisciplinares, incluida la enfermería como pilar fundamental de vigilancia y cuidados del AV, se han tenido que ir adaptando a la nueva situación, ofreciendo cuidados más específicos según las necesidades de cada paciente y del tipo de AV.

El mal funcionamiento del AV y el ser portador de un catéter tunelizado permanente (CT), se asocia a un aumento de la morbi-mortalidad y del coste en el mantenimiento del AV en estos enfermos. Las complicaciones del AV son la primera causa de ingreso hospitalario, suponiendo el mantenimiento del mismo un coste muy elevado para el sistema sanitario $^{3,4}$.

En el caso de los CT este coste aumenta no sólo por las complicaciones propias del AV, sino por las características de uso el propio CT: aumento del tiempo necesario para realizar los procesos de conexión y desconexión por parte del personal de enfermería, mayor gasto en material fungible, etc.

Actualmente, es indudable el hecho de que las unidades de HD tienden a la necesidad de ofrecer un servicio cada vez más orientado a la satisfacción del paciente, alcanzando mejores cotas de Calidad Asistencial y ofreciendo una Mejora Continua ${ }^{5}$, en el que el seguimiento del AV es un indicador necesario y evidente de mejora en la calidad del tratamiento.

Ante el aumento importante en nuestra unidad de pacientes portadores de CT nos planteamos la necesidad de la monitorización sistemática de su evolución. Se creó una base de datos específica con el fin de registrar on-line todas aquellas complicaciones derivadas de la utilización de los CT. 


\section{Objetivos}

1. Recoger todas aquellas complicaciones asociadas a la colocación y uso de CT centrales para HD en nuestra área durante dos años.

2. Realizar una estimación del coste que suponen los CT, desde su implantación, hasta su retirada, incluyendo todos los procedimientos necesarios para su correcto funcionamiento.

3. Comparar el coste de los procesos de conexión y desconexión de los CT frente a las FAV en nuestra unidad.

\section{Material y Métodos}

Durante dos años se realizó un estudio prospectivo descriptivo de 116 CT para HD, utilizados en nuestro centro hospitalario y en el centro periférico de este hospital. Todos los CT se colocaron de forma eco-dirigida y con radioscopia en el servicio de radiología vascular (RX) y fueron utilizados exclusivamente para HD. Los tipos de CT estudiados fueron (15) Quinton-Mahurkar®, (97) De BARD-Hemaglide® y (4) Canon Arrow ${ }^{\circledR}$, siendo los lugares de ubicación: yugular derecha (105), femoral derecha (4), yugular izquierda (3), subclavia izquierda (2), femoral izquierda (1) y subclavia derecha (1).

Los pacientes portadores de CT fueron 97, de los cuales el $68,05 \%$ eran hombres y el $31,95 \%$ mujeres, con una edad media de 66,56 (rango 89,7328,8) años. La etiología de la IRCT fue: diabetes mellitus $(23,3 \%)$, vasculopatía isquémica $(21,9 \%)$, nefritis intersticiales $(13,7 \%)$, poliquistosis $(6,8 \%)$, glomerulonefritis $(6,8 \%)$, vasculitis $(4,1 \%)$, mieloma o tumor $(4.1 \%)$ y otras $(2.7 \%)$.

El seguimiento del CT se realizó desde su colocación, hasta su retirada por fallecimiento, malfunción, infección, salida accidental, cambio de técnica o funcionamiento de otro acceso vascular.

Para el registro de datos se creó una base de datos tipo Access diseñada específicamente para el estudio, en la que se incluyeron los siguientes parámetros: datos de filiación del paciente, fecha de colocación, tipo, lugar de ubicación, fecha y motivo de retirada, incidencias, infecciones y tratamiento del CT. Se uti- lizó la historia informática del hospital Nefrosoft ${ }^{\circledR}$ para recoger los datos generales del estudio.

La manipulación del CT por parte de enfermería se ha realizado según protocolo validado por la comisión de calidad del centro hospitalario.

Para calcular el coste total de los CT en nuestra área, se asignó un gasto para cada procedimiento: colocación, retirada, conexión-desconexión y las manipulaciones necesarias para el mantenimiento del CT, incluyendo el precio del material fungible y el tiempo del personal sanitario, en base a los costes proporcionados por los diferentes servicios hospitalarios.

Los resultados se expresan como media \pm desviación típica. El análisis utilizado es según los casos: comparación de medias por ANOVA, Chi cuadrado para proporciones, análisis de supervivencia de KaplanMeier y Regresión de Cox. Se consideró significativo una $p<0.05$. El programa estadístico usado fue el SPSS 12.00 .

\section{Resultados}

Durante 24 meses se implantaron 116 CT en 97 pacientes en HD. En el momento actual siguen funcionantes $41 \mathrm{CT}$ y se han retirado 75 . El número de CT retirados por malfunción fueron 6 , todos debido a trombosis. El resto se retiró con buen funcionamiento por uso de FAV (37), comienzo en DP (9), infección (6), extrusión ( $5 ; 3$ en el mismo paciente), trasplante (2), recuperación de función renal (1), rotura (1) y fallecimiento con CT permeable (8).

Se recogieron 59 complicaciones relacionadas con el flujo y en todas ellas fue necesaria la administración de urokinasa. En 45 casos se solucionó el problema y se continuó el tratamiento con $\mathrm{HD}$, en 14 ocasiones fue necesaria la revisión del CT en RX; 8 fueron recolocados y 6 retirados y sustituidos por un CT nuevo.

La tasa de infección de CT fue 1,66 infecciones por cada 1.000 días de permanencia del CT. Se registraron 8 infecciones sistémicas, 7 del orificio de salida y 3 del túnel. Todas ellas se trataron con antibiótico por vía intravenosa. Se tomaron 18 cultivos: 7 negativos, dos positivos a SAMR llevándose a cabo las 
medidas de aislamiento aconsejadas por medicina preventiva. En total, se administraron 59 dosis de vancomicina, 18 de gentamicina, 4 de amoxicilina/clavulamico, 2 de cefazolina y 1 de ceftazidima. En 6 ocasiones fue necesaria la sustitución del CT por infección no recurrente. Las curas realizadas por enrojecimiento del orificio de salida se realizaron el $94 \%$ de los casos con cloruro sódico al $20 \%$ y el 6 $\%$ con antibiótico por vía tópica (Bactroban®).

La supervivencia media acumulada de los CT según

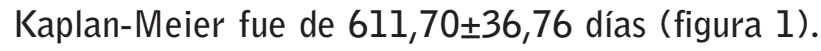

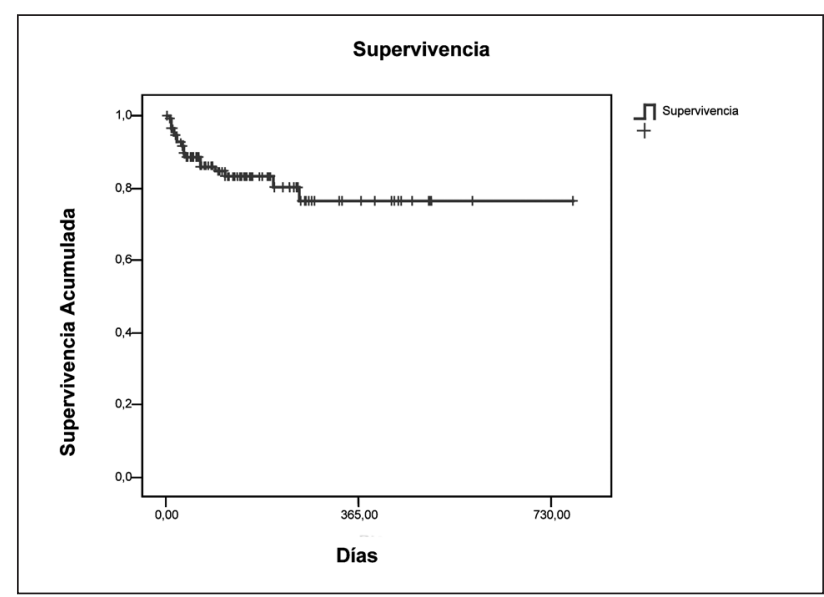

Figura 1. Supervivencia (Kaplan-Meyer) global de los catéteres en tiempo de seguimiento. T. Medio de seguimiento: $611.70 \pm 36.76$ días

Comparamos los dos grupos de CT más utilizados y observamos que la supervivencia es mayor en los Bard Hemglide $\AA \quad 605,66 \pm 39,69$, que en los Quinton-Mahurkar ${ }^{\circledR} 466,57 \pm 32,21 \quad(p<0,0489)$, aunque debemos recordar que la muestra no es homogénea y el estudio no aleatorizado, por lo que no se pueden extrapolar conclusiones sobre la superioridad de uno u otro. El tiempo de permanencia del CT según sexo fue mayor en los hombres $609,75 \pm 38,03$ vs $476,07 \pm 52,36$ días en mujeres. El análisis comparativo de la permeabilidad por localización del CT, tramos de edad, presencia de diabetes y etiología de la IRCT no mostró diferencias significativas. Sin embargo, la supervivencia del CT en los pacientes con nefropatía de origen vascular fue significativamente menor que la del resto $(293,17 \pm 46,9$ vs $642 \pm 39,08$ días, $p<0,009$ ). El número de días de funcionamiento de los CT según el motivo de retirada fue $17 \pm 3,9$ en las salidas accidentales, $43 \pm 36$ en trombosis no reparables y $79 \pm 36,8$ en las infecciones no recurren- tes (figura 2). Por último relacionamos las causas de retirada del CT y la edad y obtuvimos que la media de edad por infección no recurrente fue $49,25 \pm 18,82$, en salidas accidentales $76,20 \pm 9,80$ y en trombosis no reparables $74,78 \pm 5,13$.

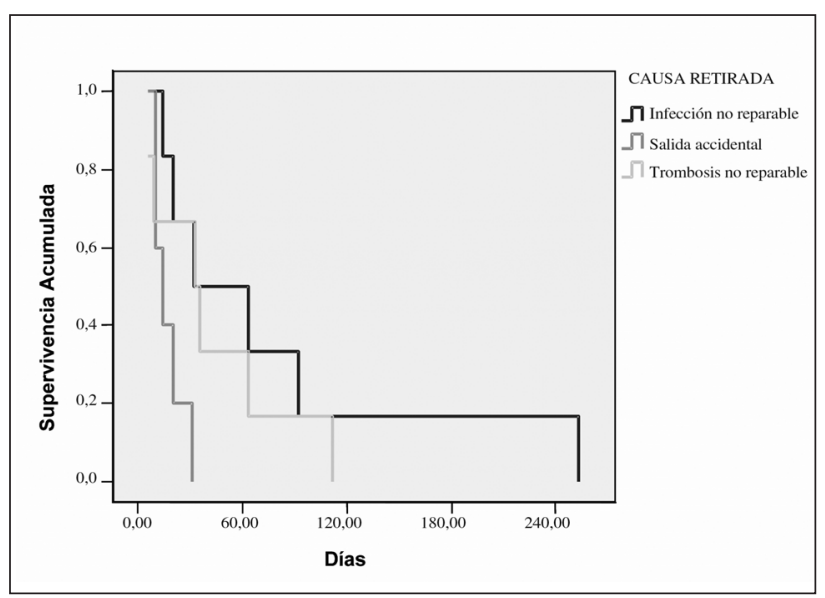

Figura 2. Supervivencia (Kaplan-Meyer) global de los catéteres según motivo de retirada

El gasto total que supusieron los CT en nuestro área fue de 203.354,07 $€$. El gasto mes/catéter fue $563,26 €$ incluyendo complicaciones (tabla 1 ). Los gastos que suponen una conexión-desconexión de CT vs FAV sin complicaciones son: 15,66 vs $6,376 €$ en FAV.

\begin{tabular}{lcccc} 
PROCEDIMIENTOS & Unidades & Coste & $\begin{array}{c}\text { Coste total } \\
\text { unidad }\end{array}$ & Por mes \\
\hline COLOCACIÓN & 116 & 871,2 & $101.059,20$ & 279,94 \\
\hline RETIRADA & 75 & 284 & $21.300,00$ & 59,00 \\
\hline REVISIÓN RX & 14 & 284 & $3.976,00$ & 11,01 \\
\hline UK DOSIS & 59 & 28,85 & $1.702,15$ & 4,72 \\
\hline CURAS LOCAL & 594 & 1,9 & $1.128,60$ & 3,13 \\
\hline ANTIBIÓTICOS & 84 & 8,146 & 684,26 & 1,90 \\
\hline CONEXIÓN-DES & 4693 & 15,661 & $73.497,07$ & 203,61 \\
\hline MESES-CATÉTER & 361,0 & & & \\
\hline TOTAL & & & $203.354,07$ & 563,26 \\
\hline
\end{tabular}

Tabla 1. Resumen de los cálculos de elementos de coste y coste total por seguimiento global y por mes catéter

\section{Discusión}

Este estudio describe nuestra experiencia en la utilización de CT permanentes y, a diferencia de otras publicaciones previas ${ }^{6}$, se ha utilizado una metodolo- 
gía de investigación con un seguimiento individual prospectivo en una base de datos creada específicamente para el mismo. Este diseño asegura el registro exhaustivo de cada evento en el momento que se produce y evita los sesgos de los registros y estudios retrospectivos. La existencia de una historia electrónica ha facilitado el seguimiento de estos accesos.

En muchos casos los CT son el último acceso para algunos enfermos, lo que supone un reto para los profesionales, que deben esforzarse en conseguir disminuir el número de complicaciones, aumentar la supervivencia del AV, mejorar la calidad de vida del enfermo y disminuir los costes asociados al uso de $\mathrm{CT}^{7}$. Hay estudios que demuestran que los pacientes portadores exclusivamente de $\mathrm{CT}$, tienen una tasa de morbi-mortalidad más elevada, así como un coste en el manejo y mantenimiento del AV mayor que el de las FAV autólogas3.

En nuestro estudio los CT presentan un funcionamiento satisfactorio con una supervivencia suficiente en la mayoría de los casos hasta que ya no es necesario el CT poniendo de manifiesto la utilidad de los $\mathrm{CT}$ en pacientes que necesitan un AV inmediato por diferentes causas. La supervivencia media acumulada fue de $611.70 \pm 36.76$ días. Encontramos diferencias entre la supervivencia de Bard Hemglide $₫$ vs Quinton-Mahurkar, aunque debemos recordar que el estudio no se diseño con este objetivo, la muestra no es homogénea en ambos subgrupos y el estudio no es aleatorizado, por lo que no se pueden extrapolar conclusiones sobre la superioridad de uno u otro. El estudio multivariante no encontró diferencias por edad, sexo, diabetes o la causa de colocación del CT. Si existía diferencia cuando comparábamos la supervivencia según etiología de la IRCT, siendo menor la supervivencia en los enfermos con patología de origen vascular.

Una de las complicaciones más serias es la infección, tras revisar las series publicadas podemos encontrar unos índices de infección del 1 al 20\%8. En nuestros resultados podemos destacar la baja tasa de infección, 1.66 infecciones por cada 1000 días de permanencia del CT, siendo el papel de los cuidados de enfermería imprescindibles para la consecución de estos resultados7,8. Hemos detectado que los pa- cientes en los que el motivo de retirada de CT ha sido la infección tienen una edad media menor que aquellos en los que se retiró por trombosis o extrusión. Hemos detectado un índice de extrusión del CT elevado ( 5 casos) pero que viene asociado a las características del paciente (tres en un mismo paciente). En estos casos, la duración del CT es la menor de todas.

Las principales causas de retirada del CT han sido el fin de utilización del mismo, por disponer de otro acceso definitivo (fístula o vuelta a DP). La tasa de infecciones y trombosis que no pueden resolverse manteniendo el mismo catéter ha sido muy baja. Nuestros datos cumplen los objetivos propuestos en las Guías de Acceso Vascular de la SEN-SEDEN?.

A pesar de la importancia del AV en HD el coste del cuidado del mismo no ha sido estudiado con detalle. Un análisis del coste de forma prospectiva en pacientes incidentes se ha hecho en Canadá 2005, el coste medio del cuidados del AV por paciente/año en riesgo de mantenimiento de $\mathrm{CT}$ fue de $5.278 €$, ligeramente inferior al obtenido en nuestro estudio $6.760 €^{3}$. El cuidado del acceso vascular es el responsable de una proporción importante de coste de cuidados de los pacientes en HD.

En nuestro estudio encontramos una diferencia importante entre el gasto (más del doble) que suponían los procedimientos de conexión-desconexión de los CT frente a las FAV. Hay estudios sobre el tema que muestran que el precio del material fungible utilizado en estos procedimientos, es tres veces mayor en los CT.

\section{Conclusiones}

1. La atención protocolizada y el registro informatizado exhaustivo nos ha llevado a una mayor concienciación ante los problemas de los CT y como resultado la reducción del número de complicaciones. EI CT permanente con túnel subcutáneo es una buena alternativa como AV.

2. En nuestra experiencia, la tasa de retirada por complicaciones del CT es escasa, cumpliendo los objetivos propuestos en las Guías de Acceso Vascular de la SEN-SEDEN. 
3. El uso y mantenimiento de los CT en nuestra área supone un gasto muy importante para el sistema sanitario

4. La conexión-desconexión de los catéteres frente a las fístulas supone algo más del doble de gasto.

\section{Bibliografía}

1. Domingo Cebrián E, Alonso Salamanca J, González Molina $\mathrm{J}$ et al. Catéteres en las Unidades de Hemodiálisis una realidad que no puede ser ignorada. En: Libro de Comunicaciones deI XXIX Congreso de la Sociedad Española de Enfermería Nefrológica; Tenerife 2004. Barcelona: HOSPAL; 2004. p. 320-321.

2. De la Vara Almonacid JA. Clasificación de los Orificios de Entrada de los Catéteres Permanentes para Hemodiálisis. En: Libro de Comunicaciones del XXX Congreso de la Sociedad Española de Enfermería Nefrológica. Málaga 2005. Barcelona: HOSPAL; 2005. p. 102-105.

3. Manns B, Tonelli M, Yilmaz S et al. Establishment and Maintenance of Vascular Access in Incident Hemodialysis Patients: A Prospective Cost Analysis. J Am Soc Nephrol. 2005; 16:201-209.

4. Tordoir HM J, Miccley V. Recomendaciones Europeas para el Acceso Vascular: Algoritmos Clínicos sobre el Acceso Vascular para Hemodiálisis. EDTNA / ERCA Journal 2003; XXIX (3): 133-139.
5. Hernández Meca ME, Ochando García A, Mora Canales $J$ et al. Satisfacción del Paciente en una Unidad de Hemodiálisis: Objetivo de Calidad Asistencial en Enfermería. En:Libro de Comunicaciones del XXIX Congreso de la Sociedad Española de Enfermería Nefrológica. Tenerife 2004. BarceIona: HOSPAL; 2004. p. 139-140.

6. López Ramón I, Muro Suescun B, Azcona Ezcurra $M$ et al. Cuatro años de Experiencia con 20 Catéteres Twin-Cath: Indicaciones, Supervivencia y Prestaciones. En: Libro de Comunicaciones del XXII Congreso de la Sociedad Española de Enfermería Nefrológica. Santander 1997 Barcelona: HOSPAL; 1997. p. 18-24.

7. Crespo Montero R, Rivero F, Contreras MD. Análisis de las Complicaciones y duración de los Catéteres Temporales para Hemodiálisis. En: Libro de Comunicaciones del XXII Congreso de la Sociedad Española de Enfermería Nefrológica. Santander 1997 Barcelona: HOSPAL; 1997.

8. Polaschegg HD, Sodemann K, Feldmer B. Aumento de la permeabilidad, Seguridad y Eficacia en el Coste de los Catéteres. EDTNA / ERCA Journal 2002; XXVIII (1): 29-33.

9. Rodríguez Hernández JA, González Parra E, Gutierrez Julian JM et al. Guías del Acceso Vascular de Hemodiálisis. Nefrología. XXV. Suplemento 1. 2005. 